\title{
Origin of Sepsis Associated with the Short-Term Mortality of Patients: A Retrospective Study Using the elCU Collaborative Research Database
}

\author{
Qinglin $\mathrm{Li}^{1}$ \\ Yingmu Tong' \\ Hai Wang' \\ Jie Ren' \\ Sinan Liu ${ }^{1,2}$ \\ Tong Liu' \\ Kai Qu' \\ Chang Liu ${ }^{1,2}$ \\ Jingyao Zhang $\mathbb{D}^{1,2}$
}

'Department of Hepatobiliary Surgery, The First Affiliated Hospital of Xi'an jiaotong University, Xi'an, Shaanxi, 71006I, People's Republic of China; ${ }^{2}$ Department of SICU, The First Affiliated Hospital of Xi'an Jiaotong University,

Xi'an, Shaanxi, 7I006I, People's Republic of China
Correspondence: Chang Liu; Jingyao Zhang The First Affiliated Hospital of Xi'an Jiaotong University, 277 Yanta West Road, Xi'an,

7 I006I, People's Republic of China

Tel +86-29-85323900

Fax +86-29-85324642

Email liuchangdoctor@163.com;

youl2ouy@l63.com
Objective: The aim of this study was to compare the clinical characteristics and short-term mortality of patients with abdominal and pulmonary sepsis.

Design: Retrospective cohort study.

Setting: Adult intensive care units (ICUs) at tertiary hospitals.

Participants: Adult ICU patients from 2014 to 2015 in the eICU Collaborative Research Database.

Interventions: In univariate analysis, we compared the differences in the characteristics of patients in each group. Logistic regression models were used to evaluate the relationships between primary site of sepsis and short-term prognosis.

Primary and Secondary Outcome Measures: Hospital and ICU survival.

Results: The final dataset included 7023 pulmonary and 2360 abdominal sepsis patients, who accounted for $74.84 \%$ and $25.16 \%$, respectively. We compared the results of the baseline characteristics, vital signs and laboratory indicators between the two groups. In the logistic regression models, we found that the hospital and ICU mortality of patients with abdominal sepsis was higher than that with pulmonary sepsis $(\mathrm{p}<0.05, \mathrm{OR}=1.15, \mathrm{p}<0.05$, $\mathrm{OR}=1.19$, respectively), although these results were no longer significantly after adjustment for confounders, but in the subgroups with SOFA score $\geqq 8$, the adjusted hospital mortality rate of patients with abdominal sepsis was 1.30 times higher than that of patients with pulmonary sepsis $(\mathrm{p}<0.005, \mathrm{OR}=1.30,95 \%$ CI 1.09-1.55), while there was no significant difference in the subgroups that SOFA score $<8$.

Conclusion: In the patients with SOFA score $\geqq 8$, the adjusted hospital mortality of patients with abdominal sepsis was higher than patients with pulmonary sepsis.

Keywords: sepsis, pulmonary, abdominal, eICU-CRD, ICU

\section{Introducion}

Sepsis is a complex and acute disease, which develops from the host's maladjustment with infection. It is associated with high risk of death and acute organ dysfunction. This syndrome needs urgent treatment, and it is important to acquaintance its characteristics. The incidence of sepsis is very high, which is still one of the main causes of death worldwide. ${ }^{1}$

The most common causes of sepsis are bacterial infections of the lungs and abdomen. ${ }^{2}$ According to epidemiological data, the lung is the most common site of infection $(68 \%)$ in the sepsis, followed by the abdomen $(22 \%){ }^{3}$ Previous studies have shown that the site of infection may play an independent role in the survival of 
patients with sepsis. ${ }^{4}$ Some previous studies have shown that the mortality of sepsis associated with pneumonia was higher among critically ill patients. ${ }^{5}$ There was one study aimed at the long-term outcome and quality of life in pulmonary sepsis and abdominal sepsis. ${ }^{6}$ However, existing research on the short-term outcome of sepsis according to the primary site was contested and information about difference between abdominal sepsis and pulmonary sepsis was still limited.

Sequential Organ Failure Assessment or SOFA score was developed to assess the acute morbidity of critical illness at a population level and has been widely validated as a tool for this purpose across a range of health-care settings and environments. ${ }^{7}$ Some previous studies have shown that SOFA score $\geq 8$ was an independent risk factor for some diseases like severe infective endocarditis in intensive care unit. ${ }^{8,9}$ Another study shows that SOFA score $\geq 8$ can be used as an indicator of ICU admission during influenza pandemic when ICU beds are relatively insufficient. ${ }^{10}$ Hence, SOFA score $>8$ was used as a cutoff point in some clinical studies, while similar studies have not been carried out in abdominal and pulmonary sepsis.

The aim of this study was to compare the clinical characteristics and short-term outcome of patients with abdominal and pulmonary sepsis.

\section{Methods}

The present study is reported in accordance with the Reporting of Studies Conducted using the Observational Routinely Collected Health Data (RECORD) statement. ${ }^{11}$

\section{Setting}

The eICU Collaborative Research Database (eICU-CRD) is a multi-center intensive care unit (ICU) database with over 200,000 admissions to ICUs developed by Philips Healthcare across the United States. The data contains unidentified data on more than 200,000 ICU admissions from 208 different ICUs in the United States between 2014 and 2015. ${ }^{12}$ The eICU-CRD including information about patient admission, discharge, demographics, physiologic data (from bedside monitors, ventilators, and other devices), laboratory results, severity scores, procedures, and medications. The data can be publicly used after registration, and many applications have been developed using this database, including the development of machine learning algorithms, decision support tools, and clinical research. ${ }^{13,14}$

\section{Ethical Approval}

This study has been approved by the Institutional Review Board (IRB) of the Massachusetts Institute of Technology (MIT). After successfully accomplishing the National Institutes of Health's (NIH) online training course and the Protection of Human Research Participants Examination, we had the access to extract data from eICUCRD. All data were extracted by the corresponding author (Certification ID: 28572693). This study was also reviewed by IRB of the First affiliated hospital of Xi'an Jiaotong University and waived the requirement for ethics approval. Given that all patients in this database were deidentified, informed consent was waived.

\section{Study Population}

Patients with abdominal and pulmonary sepsis in the eICU-CRD database met the inclusion criteria. For patients who had been admitted to ICU for many times, only the first admission record was kept. The exclusion criteria included: (1) age under 18 years old (2) without ICU and hospital dead/survival data.

\section{Data Extraction and Management}

The following variables were collected on the first day of admission: age, sex, admission weight, admission height, Sequential Organ Failure Assessment (SOFA) score, Charlson comorbidity score, vasopressors use, renal replacement therapy (RRT) and mechanical ventilation in the first 24 hours of admission, and length of stay (LOS) in hospital. The BMI was calculated based on height and weight. The SOFA score were calculated on the first 24 hours after the admission. We also used the above data to diagnose whether the patient had organ dysfunction through the diagnostic criteria of MODS. ${ }^{9}$ If a variable was measured more than once in the first 24 hours, the value associated with the most severe degree of the disease was used. For example, the lowest mean blood pressure (MBP) and Glasgow Coma Scale (GCS) score reported in the first 24 hours were used in the study. In addition, dates of birth for patients aged over 89 in the database were shifted to obscure their true age: these patients appear in the database with ages of over 89 years, but the median age of these patients was 92.5 years old, so we shifted the age of these patients to 92.5 years old. Variables with more than $40 \%$ of values missing were excluded from the analysis. For variables with missing values outside of this range, we used multiple imputation (MI), based on five 
replications and a chained equation approach method in the R STUDIO MI procedure, to account for missing data. $^{15}$

\section{Outcomes}

The primary and secondary objective of the study were the hospital and ICU mortality.

\section{Statistical Analysis}

First, univariate analysis was used to compare all of the variables. If the data adequated a normal distribution and the variance was equal variances, the data are expressed as the mean \pm standard deviation, and Student's $t$-test was used for comparisons. If the variance was not equal variances, then Welch test was used for the comparisons. If none of the above requirements were met or the data were not continuous variables, then the data are described as the median and interquartile range (IQR), and the Wilcoxon rank-sum test was used for comparisons. Categorical variables are presented as numbers and percentages and were compared by Pearson's chi-square test or Fisher's exact test as appropriate.

Propensity score matching (PSM) was performed to minimize the influence of confounding factors on selection bias. The propensity scores were elicited from matched patients in a 1:1 ratio with greedy nearest neighbor matching algorithms without replacement. Since there were more than 7000 patients with pulmonary sepsis and about 2000 patients with abdominal sepsis, pulmonary sepsis group was used as the treatment group and abdominal group was considered control group. The caliper, also known as the match tolerance, be used was 0.001 . We adjusted for age, gender, BMI, ethnicity, region, Charlson comorbidity score and SOFA, but in the subgroup analysis we did not adjust the SOFA score. Multivariate analyses were adjusted for the possible variables that may affect the prognosis of patients to determine the relationship between primary site of sepsis and ICU and hospital mortality. We tested the collinearity of the variables included in the statistical analysis, and found that the variance inflation factor (VIF) of all variables was $<3$; hence, there was no statistical collinearity in the included variables. Variables with $\mathrm{p}<0.10$ in univariate analysis were included in the Logistic regression model as confounders to determine whether primary site was the independent risk factor of the ICU and hospital survival rates.

SPSS (version 25.0; IBM, Armonk, NY) and EmpowerStats (version 2018-05-05, copyright 2009
X\&Y Solutions, Inc) were used for data analysis; a twotailed $\mathrm{p}<0.05$ was considered statistically significant. R STUDIO was used for PSM to adjusting for confounding factors.

\section{Results}

In the eICU-CRD, 23,136 patients were diagnosed with sepsis, and 11,763 patients with abdominal and pulmonary sepsis. After excluding the patients who did not enter the ICU for the first time, 10,075 patients remained, including 2 patients who were younger than 18 years old. Finally, patients without ICU and hospital dead/survival data and with malignant entities were excluded and deleted. The final dataset included 7023 pulmonary and 2360 abdominal sepsis patients, accounting for $74.84 \%$ and $25.15 \%$, respectively (Figure 1).

\section{Clinical Characteristics}

The patient characteristics obtained within the first 24 hours in the ICU are presented in Table 1. The median age of the pulmonary sepsis patients was higher than in the abdominal sepsis group (69 vs 67, $\mathrm{p}<0.001$ ). The proportion of males in the group of patients with pulmonary sepsis was lower than that in the abdominal sepsis group (46.4\% vs $50.6 \%$, p $<0.001)$. The group with pulmonary sepsis had a lower BMI than the abdominal sepsis group $(\mathrm{p}=0.011)$. After confounder adjustment, these variables were turned into no significant difference (Table S1).

The abdominal sepsis group had a higher ICU mortality, but their LOS in ICU was shorter than the pulmonary group ( $\mathrm{p}<0.001, \mathrm{p}<0.001$, respectively), after confounder adjustment, this result did not change $(\mathrm{p}=0.008, \mathrm{p}<$ 0.001). However, there was no significant difference between the two groups in regard to hospital mortality or LOS in the hospital ( $\mathrm{p}>0.005$, respectively). About the SOFA scores, although the analysis showed statistical differences, the medians were the same $(\mathrm{p}<0.001$, respectively).

The results of the treatment are shown in Table 2. The patients with abdominal sepsis had a higher rate of using vasoactive drugs than the pulmonary group $(32.7 \%$ vs. $25.4 \%, p<0.001$, while in terms of mechanical ventilation, the conclusion is just the opposite $(26.6 \%$ vs $31.9 \%, \mathrm{p}<0.001)$. There was no difference in renal replacement therapy $(\mathrm{p}=0.124)$. After confounder adjustment, those results did not change (Table S2).

The most common comorbidities were cardiac arrhythmia (1391, 14.4\%), fluid electrolyte disorders (1275, 


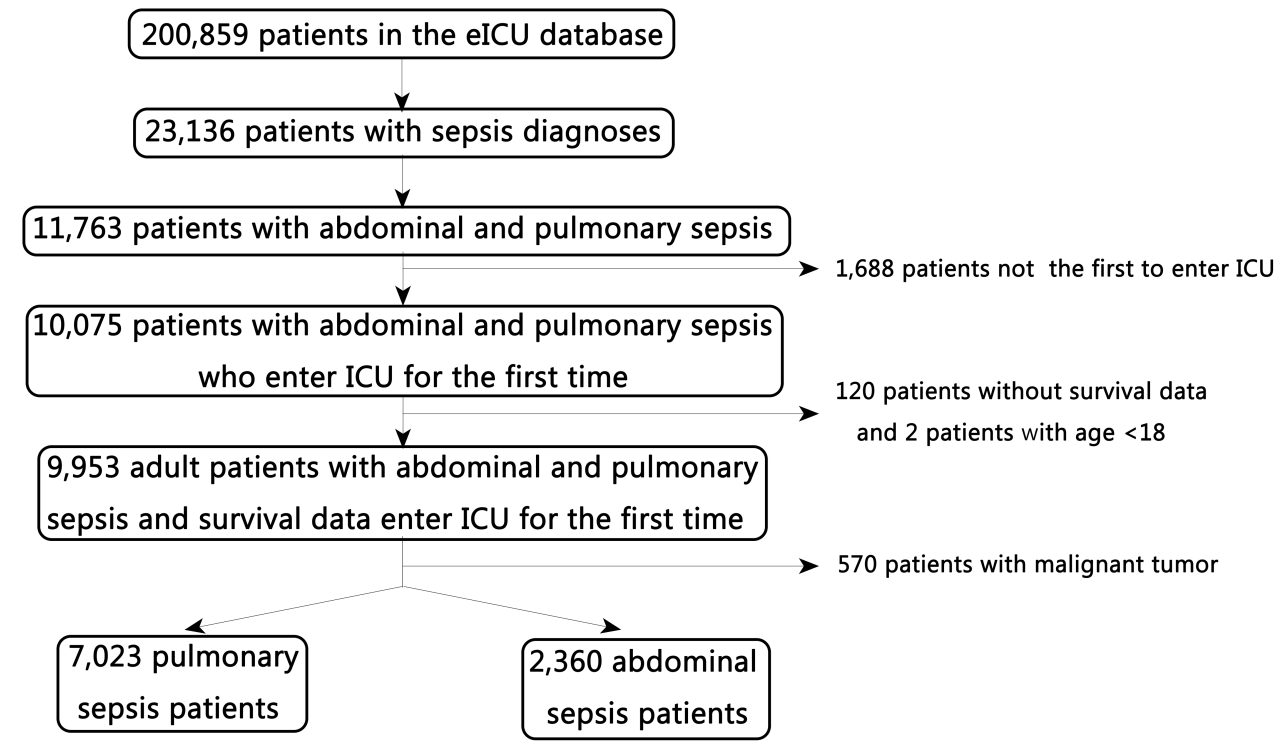

Figure I Flowchart of the patient selection process in this study.

$13.2 \%)$, chronic pulmonary $(1159,12.0 \%)$, renal failure (910, 9.4\%), and congestive heart failure (906, 9.4\%). Among them, fluid electrolyte, chronic pulmonary and congestive heart failure had significant differences in the constituent ratio $(11.7 \%$ vs $18.5 \%, 14.8 \%$ vs $4.6 \%, 11.2 \%$ vs $4.5 \%, \mathrm{p}<0.001$, respectively). The results are shown in Table S3.

\section{Vital Signs and Laboratory Values}

Vital signs and laboratory values during the first 24 hours are presented in Table 3. In terms of vital signs, the heart rate of patients with pulmonary sepsis was lower than that of patients with abdominal sepsis $(\mathrm{p}<0.001)$, while systolic blood pressure, diastolic blood pressure and mean arterial pressure were higher than those with abdominal sepsis $(\mathrm{p}<0.001, \mathrm{p}=0.005, \mathrm{p}<0.001$, respectively). The respiratory rate and body temperature of patients with pulmonary sepsis were higher than those of the other group ( $\mathrm{p}<0.001, \mathrm{p}<0.001$, respectively). After confounder adjustment, there was no significant difference in heart rate between the two groups.

As for the laboratory values, if the infection was located in the lung, the patients had lower white blood cell (WBC) and platelet count (PLT) values $(\mathrm{p}<0.001, \mathrm{p}=0.021$, respectively), and after confounder adjustment, these variables still showed significant difference. There was no significant difference in their hemoglobin (HGB) values. In terms of liver and kidney function of pulmonary sepsis group, the bilirubin (BIL), creatinine (CRE) and blood urea nitrogen (BUN) values were lower than those in the abdominal sepsis group ( $<<0.001, \mathrm{p}<0.001, \mathrm{p}<0.001$, respectively), after confounder adjustment, the results did not change. While in the albumin (ALB) values the analysis showed statistical differences, the medians were the same ( $p<0.001$ ), and after confounder adjustment, there was no significant difference in ALB between the two groups. In terms of coagulation indexes, activated partial thromboplastin time (APTT), international normalized ratio (INR) and prothrombin time (PT) in patients with abdominal sepsis were higher than those in patients with pulmonary sepsis $(\mathrm{p}<$ $0.001, p<0.001, p<0.001$, respectively). After confounder adjustment, APTT was no longer statistically significant, and the other two indicators were still higher in the abdominal sepsis group. For the electrolyte index, sodium $(\mathrm{Na})$ in the two groups had the same median, but their distributions were statistically different $(\mathrm{p}<0.001)$, while for chlorine $(\mathrm{Cl})$, the pulmonary sepsis patients had lower values $(\mathrm{p}<0.001)$. There was no significant difference in potassium between the two groups. After confounder adjustment, only $\mathrm{Cl}$ still showed significance between the groups (Table S4).

Finally, for the blood gas analysis part, fraction of inspiration $\mathrm{O}_{2}\left(\mathrm{FiO}_{2}\right)$, partial pressure of carbon dioxide in artery $\left(\mathrm{PaCO}_{2}\right), \mathrm{pH}$, and bicarbonate, the values of pulmonary sepsis patients were higher than that of abdominal sepsis patients $(\mathrm{p}<0.001, \mathrm{p}<0.001, \mathrm{p}<0.001, \mathrm{p}<$ 0.001 , respectively), while the conclusion for alveolar oxygen partial pressure $\left(\mathrm{PaO}_{2}\right)$, anion gap $(\mathrm{AG})$ and lactate (LAC) was the opposite $(\mathrm{p}=0.004, \mathrm{p}<0.001, \mathrm{p}<0.001)$. 
Table I Univariate Analysis of Baseline Characteristics by Sepsis Position Category

\begin{tabular}{|c|c|c|c|}
\hline Variables & $\begin{array}{c}\text { Pulmonary } \\
\text { Sepsis } \\
(n=7023)\end{array}$ & $\begin{array}{l}\text { Abdominal } \\
\text { Sepsis } \\
(n=2360)\end{array}$ & p values \\
\hline Age, yr, median(IQR) & $69(57,80)$ & $67(55,78)$ & $<0.001$ \\
\hline Gender, n(\%) & & & $<0.001$ \\
\hline Female & 3761 (53.6\%) & 1165 (49.4\%) & \\
\hline Male & 3261 (46.4\%) & II 95 (50.6\%) & \\
\hline Ethnicity, n(\%) & & & 0.001 \\
\hline Caucasian & $5598(80.5 \%)$ & 1870 (79.8\%) & \\
\hline African American & 609 (8.8\%) & $158(6.7 \%)$ & \\
\hline Asian & $120(1.7 \%)$ & $54(2.3 \%)$ & \\
\hline Hispanic & 227 (3.3\%) & 97 (4.1\%) & \\
\hline Native American & $62(0.9 \%)$ & $26(1.1 \%)$ & \\
\hline Other/Unknown & $335(4.8 \%)$ & $139(5.9 \%)$ & \\
\hline BMI $\left(K g / m^{2}\right)$ & & & $<0.001$ \\
\hline$<19$ & $612(9.0 \%)$ & 145 (6.4\%) & \\
\hline $19-24$ & $1743(25.6 \%)$ & $573(25.2 \%)$ & \\
\hline$>24$ & 4449 (65.4\%) & 1555 (68.4\%) & \\
\hline Region, $n(\%)$ & & & $<0.001$ \\
\hline Midwest & 2877 (45.0\%) & 785 (35.7\%) & \\
\hline Northeast & $410(6.4 \%)$ & $182(8.3 \%)$ & \\
\hline South & 1394 (21.8\%) & $583(26.5 \%)$ & \\
\hline West & $1714(26.8 \%)$ & $651(29.6 \%)$ & \\
\hline Charlson comorbidity score & $0(0,1)$ & $0(0,1)$ & $<0.001$ \\
\hline qSOFA & $2(1,3)$ & $2(1,2)$ & $<0.001$ \\
\hline SOFA & $5(3,8)$ & $5(3,9)$ & $<0.001$ \\
\hline LOS ICU, d, median(IQR) & $2.54(1.33,5.17)$ & $2.13(1.17,4.00)$ & $<0.001$ \\
\hline LOS Hospital, d, median(IQR) & $7.08(4.21,11.92)$ & $7.08(3.85,12.96)$ & 0.070 \\
\hline ICU mortality, n(\%) & $887(12.6 \%)$ & $346(14.7 \%)$ & $<0.001$ \\
\hline Hospital mortality, n(\%) & $137 \mid(19.5 \%)$ & $515(21.8 \%)$ & 0.813 \\
\hline
\end{tabular}

Abbreviations: IQR, interquartile range; BMI, body mass index; SOFA, Sequentia Organ Failure Assessment; LOS, long of stay.

After confounder adjustment, the $\mathrm{PaO}_{2}$ turned into no significant difference (Table S4).

\section{Logistic Regression Model}

We imported variables with $\mathrm{p}$ values $<0.10$ in univariate analysis into Logistic regression model as independent variables after testing the collinearity of the variables. When the patient's diagnosis applied as a classification

Table 2 Univariate Analysis of Treatments by Sepsis Position Category

\begin{tabular}{|l|l|l|l|}
\hline $\begin{array}{l}\text { Treatments, n, } \\
\text { (\%) }\end{array}$ & $\begin{array}{l}\text { Pulmonary } \\
\text { Sepsis } \\
(\mathbf{n}=\mathbf{7 0 2 3})\end{array}$ & $\begin{array}{l}\text { Abdominal } \\
\text { Sepsis } \\
(\mathbf{n}=\mathbf{2 3 6 0})\end{array}$ & p value \\
\hline Vasopressor & $1782(25.4 \%)$ & $772(32.7 \%)$ & $<0.00 \mathrm{I}$ \\
Ventilation & $2238(31.9 \%)$ & $628(26.6 \%)$ & $<0.00 \mathrm{I}$ \\
Dialysis & $84(1.2 \%)$ & $38(1.6 \%)$ & 0.124 \\
\hline
\end{tabular}

variable, it was associated with the ICU and hospital mortality of patients with abdominal sepsis, the OR values were $1.19(1.04,1.36)$ and $1.15(1.03,1.29)$ respectively, but after adjusted for age, gender, BMI, ethnicity, region, Charlson comorbidity score and SOFA score, the result were no longer significant (Table 4). We conjectured that there was an interaction effect in the adjusted variables, after testing the interaction effects of those variables in turn, we confirmed that there were interactions among the SOFA scores and diagnosis (Table S5). Previous studies have shown that SOFA score $\geq 8$ was an independent risk factor for some diseases. ${ }^{8,16}$ Another study shows that SOFA score can be used as an indicator of ICU admission during influenza pandemic when ICU beds are relatively insufficient. When SOFA $<8$, patients tend to have a better prognosis and prefer to stay in ICU; When SOFA $>8$, patients have lower priority in ICU due to poor prognosis. ${ }^{10}$ SOFA>8 was used as the cut-off point in some clinical studies. ${ }^{17}$ Then we subsequently divided the patients into two subgroups with SOFA score greater than 8 and less than 8 . In the subgroup with a SOFA scores $\geqq 8$, the adjusted OR values of hospital outcome were 1.30 $(1.09,1.55)$, but in the subgroup with a SOFA scores $<8$, the adjusted OR values were not significant (Table 5).

\section{Discussion}

In this retrospective study, we compared the clinical characteristics and short-term outcome of patients with abdominal and pulmonary sepsis in the eICU-CRD database. We first compared the baseline characteristics of the two groups of patients. Patients with pulmonary sepsis had a higher age and a higher proportion of female. Multivariate analysis showed that the prognosis of abdominal sepsis was worse than that of pulmonary sepsis, which was only significant in the subgroup of patients with SOFA score $>8$. The first half part of this result conflicts with some previous studies, which may be because Li et al focused on the long-term prognosis of pulmonary and abdominal sepsis, while our results focused on the shortterm prognosis. ${ }^{6}$ In our results, patients with abdominal sepsis have higher SOFA score, and the occurrence of multiple organ failure, such as liver, kidney and coagulation system failure, was more serious, which may lead to worse prognosis. In essence, sepsis is an organ dysfunction caused by an inadequate or dysregulated host response to infection. ${ }^{18}$ The primary site of infection was not only related to the disease that caused the sepsis, but was also related to the progression of the sepsis. For 
Table 3 Vital Signs and Laboratory Values by Sepsis Position Category

\begin{tabular}{|c|c|c|c|}
\hline Variables, Median (IQR) & $\begin{array}{l}\text { Pulmonary Sepsis } \\
(n=7023)\end{array}$ & $\begin{array}{l}\text { Abdominal Sepsis } \\
(n=2360)\end{array}$ & p value \\
\hline \multicolumn{4}{|l|}{ Vital signs } \\
\hline Heart rate $(\mathrm{n} / \mathrm{min})$ & $117(101,134)$ & $119(102,138)$ & 0.015 \\
\hline Respiratory rate (n/min) & $32(26,38)$ & $30(27,39)$ & $<0.001$ \\
\hline Body temperature $\left({ }^{\circ} \mathrm{C}\right)$ & $37.6(37.1,38.4)$ & $37.4(36.9,38.1)$ & $<0.001$ \\
\hline Systolic blood pressure $(\mathrm{mmHg})$ & $138(124,156)$ & $135(121,152)$ & $<0.001$ \\
\hline Diastolic blood pressure $(\mathrm{mmHg})$ & $83(7 I, 96)$ & $81(70,95)$ & 0.005 \\
\hline Mean arterial pressure $(\mathrm{mmHg})$ & $97(86,110)$ & $95(83,107)$ & $<0.001$ \\
\hline \multicolumn{4}{|l|}{ Blood routine } \\
\hline HGB (g/dL) & $11.7(10.2,13.6)$ & II.5 (9.8, I3.5) & 0.760 \\
\hline WBC (10^9/L) & $16.5(11.4,22.6)$ & $18.6(12.5,27.3)$ & $<0.001$ \\
\hline PLT (10^9/L) & $218(158,298)$ & $224(15 I, 318)$ & 0.021 \\
\hline \multicolumn{4}{|l|}{ Liver function } \\
\hline ALB (g/dL) & $2.8(2.4,3.4)$ & $2.8(2.3,3.4)$ & $<0.001$ \\
\hline BIL (umol/L) & $0.7(0.5,1.1)$ & $1.0(0.6,2.2)$ & $<0.001$ \\
\hline \multicolumn{4}{|l|}{ Kidney function } \\
\hline BUN (mmol/L) & $34(22,50)$ & $39(28,58)$ & $<0.001$ \\
\hline CRE (umol/L) & $1.34(0.93,2.20)$ & $1.68(1.07,2.85)$ & $<0.001$ \\
\hline \multicolumn{4}{|l|}{ Coagulation indexes } \\
\hline APTT(s) & $34.0(29.0,45.0)$ & $36.5(31.0,48.0)$ & $<0.001$ \\
\hline INR & I.4 (I.I, I.8) & $1.6(1.3,2.7)$ & $<0.001$ \\
\hline $\mathrm{PT}(\mathrm{s})$ & $16.2(13.8,20.8)$ & $19.0(15.0,29.2)$ & $<0.001$ \\
\hline \multicolumn{4}{|l|}{ Electrolyte index } \\
\hline $\mathrm{Cl}(\mathrm{mmol} / \mathrm{L})$ & $107(103,112)$ & $109(105,113)$ & $<0.001$ \\
\hline $\mathrm{K}(\mathrm{mmol} / \mathrm{L})$ & $4.5(4.0,5.1)$ & $4.7(4.2,5.3)$ & 0.667 \\
\hline $\mathrm{Na}(\mathrm{mmol} / \mathrm{L})$ & $14 \mid(137,144)$ & $|4|(137,145)$ & $<0.001$ \\
\hline \multicolumn{4}{|l|}{ Blood gas analysis } \\
\hline $\mathrm{FiO} 2$ & $0.80(0.50,1.00)$ & $0.60(0.36,1.00)$ & $<0.001$ \\
\hline $\mathrm{PaO} 2(\mathrm{mmHg})$ & $114(85,179)$ & $126(90,210)$ & 0.004 \\
\hline $\mathrm{PaCO} 2(\mathrm{mmHg})$ & $43.30(36.00,55.00)$ & $38.00(31.00,45.00)$ & $<0.001$ \\
\hline $\mathrm{pH}$ & $7.38(7.33,7.45)$ & $7.36(7.36,7.43)$ & $<0.001$ \\
\hline $\mathrm{SpO} 2$ & $100(99,100)$ & $100(100,100)$ & 0.230 \\
\hline AG $(\mathrm{mmol} / \mathrm{L})$ & $12(9,16)$ & $14(11,19)$ & $<0.001$ \\
\hline $\mathrm{LAC}(\mathrm{mmol} / \mathrm{L})$ & $2.9(1.7,5.2)$ & $4.0(2.2,8.6)$ & $<0.001$ \\
\hline Bicarbonate $(\mathrm{mmol} / \mathrm{L})$ & $25(22,29)$ & $23(20,26)$ & $<0.001$ \\
\hline
\end{tabular}

Abbreviations: IQR, interquartile range; HGB, hemoglobin; WBC, white blood cell count; PLT, platelet count; ALB, albumin; BIL, bilirubin; CRE, creatinine; BUN, urea nitrogen; LAC, lactate; APTT, activated partial thromboplastin time; INR, international normalized ratio; PT, Prothrombin time; AG, Anion gap; Na, sodium; Cl, chlorine; K, potassium; $\mathrm{FiO} 2$, fraction of inspiration $\mathrm{O} 2$; $\mathrm{PaCO} 2$, partial pressure of carbon dioxide in artery; $\mathrm{PaO}$, alveolar oxygen partial pressure; $\mathrm{SpO}$, pulse oxygen saturation.

example, pneumonia was the main cause of pulmonary sepsis, while abdominal sepsis was usually caused by peritonitis and perforation of abdominal organ. ${ }^{19}$ However, sometimes there were exceptions, and pulmonary infection may be complicated with abdominal sepsis. ${ }^{20}$ Although sepsis was a disease that affects the whole body, the pathophysiological response of different organs was different. $^{21}$ In the process of pulmonary sepsis, increased vascular permeability is considered to be a part of acute lung injury, which is widely recognized and accepted. It has been proven that LPS can cause severe injury of pulmonary endothelial cells and increase their permeability. $^{22,23}$ The gas exchange capacity of the alveoli would also be affected by the inflammatory exudates. ${ }^{24}$ In our results, patients with pulmonary sepsis had a higher proportion $(p<0.001)$ of using mechanical ventilation and pulmonary sepsis patients also tend to develop ARDS earlier. ${ }^{25}$ In the abdominal sepsis, increased gut permeability is more common; however, therapeutics targeting the gut have proven to be challenging to implement at the bedside. $^{26}$ A large number of pathogens in the intestine can enter the abdominal cavity and blood through the 
Table 4 Result of the Logistic Regression Analysis

\begin{tabular}{|l|l|l|}
\hline Exposure & Non-Adjusted OR, p value & Adjusted OR, p value \\
\hline ICU mortality & & \\
Pulmonary sepsis & 1.00 (Reference) & 1.00 (Reference) \\
Abdominal sepsis & $1.19(1.04,1.36), 0.0117$ & $1.06(0.91,1.24), 0.4732$ \\
Hospital mortality & & \\
Pulmonary sepsis & 1.00 (Reference) & 1.00 (Reference) \\
Abdominal sepsis & $1.15(1.03,1.29), 0.0160$ & $1.07(0.94,1.23), 0.2878$ \\
\hline
\end{tabular}

Note: Adjusted for: age, gender, BMI, ethnicity, region, Charlson comorbidity score and SOFA.

Abbreviation: ICU, intensive care unit.

Table 5 Result of the Logistic Regression Analysis in Subgroup of SOFA Scores

\begin{tabular}{|l|l|l|}
\hline Exposure & $\begin{array}{l}\text { SOFA Scores <8 Subgroup } \\
\text { OR }(\mathbf{9 5 \%} \mathbf{~ C I}), \mathbf{p} \text { value }\end{array}$ & $\begin{array}{l}\text { SOFA Scores } \geqq 8 \text { Subgroup } \\
\text { OR }(\mathbf{9 5 \%} \mathbf{C I}), \mathbf{p} \text { value }\end{array}$ \\
\hline $\begin{array}{l}\text { Hospital mortality } \\
\text { Non-adjusted }\end{array}$ & & \\
$\quad \begin{array}{l}\text { Pulmonary sepsis } \\
\text { Abdominal sepsis }\end{array}$ & 1.00 (Reference) & 1.00 (Reference) \\
Adjusted & $0.95(0.80,1.13), 0.5589$ & $1.24(1.05,1.47), 0.0131$ \\
$\quad \begin{array}{l}\text { Pulmonary sepsis } \\
\text { Abdominal sepsis }\end{array}$ & 1.00 (Reference) & 1.00 (Reference) \\
\hline
\end{tabular}

Note: Adjusted for: age, gender, BMI, ethnicity, region and Charlson comorbidity score.

damaged intestinal barrier, and this may cause bacteremia and diffuse peritonitis in sepsis when the immune system is dysfunctional. ${ }^{27}$ The abundant vascular and lymphatic system in the abdominal cavity may lead to the bacteria more easily spreading through these pathways. ${ }^{28}$ In terms of the use of vasopressor drugs and fluid resuscitation, patients with abdominal sepsis use them more frequently and have a larger fluid input, which was same in our result. This may be due to the more common Gramnegative bacteria released in abdominal sepsis. Endotoxin is more likely to cause the spasm of small blood vessels, thereby causing microcirculation disorders. ${ }^{29}$ The smaller urine output of patients with abdominal sepsis also indicated a poorer prognosis, which can be mutually confirmed by their higher ICU mortality rate.

For the second part of multivariate analysis, which was only in patients with SOFA score $>8$, the short-term prognosis of abdominal sepsis group was worse than that of pulmonary sepsis group. In previous studies, patients with SOFA $>8$ were considered to be medium or low priority according to current pandemic triage criteria. ${ }^{10}$ SOFA score was generally considered to be closely related to the prognosis of patients with sepsis, which indicated the prognosis of patients with SOFA $>8$ were worse than that of patients with SOFA $<8$, and the treatment effect of patients with a SOFA score $>8$ may not be obvious after receiving intensive care treatment. From the perspective of health economics, especially in the period of infectious diseases such as COVID-19, the rational allocation of resources was very important, on the one hand, the results of this study showed that patients with abdominal sepsis were worse in organ failure and other aspects, resulting in poor short-term prognosis than patients with pulmonary sepsis, and they may need to enter ICU earlier for intensive care treatment. On the other hand, it showed that even in patients with SOFA $>8$ also needed to be divided according to the specific source of sepsis in order to get the most appropriate treatment, their priority of entering ICU was divided into medium and low priority. At the same time, most of the existing clinical research designs on sepsis still regarded sepsis as a systemic disease. Our research suggested that the primary site of sepsis should be adjusted as a confounding factor in the future research, or the primary site of sepsis should be paid attention to in the design of research to eliminate unnecessary confounding factors. 
Our research has the following limitations. First, despite the inclusion of a large number of confounders, residual confounding due to inaccurate proxy variables or unmeasured variables cannot be ruled out. Second, we only obtained the baseline characteristic information of patients and some laboratory examination results of patients within 24 hours after admission, but did not specifically study the infection and treatment process of the patients (such as use of antibiotics, etc.), and the disparate interventions in the two groups in regard to these factors may lead to deviations in our results. In the end, our study only used the eICU-CRD and did not use external data sets for verification, which may affect the external validity of study.

\section{Conclusion}

In the patients with SOFA score $\geq 8$, the adjusted hospital mortality of patients with abdominal sepsis was higher than patients with pulmonary sepsis.

\section{Abbreviations}

ARDS, acute respiratory distress syndrome; ICU, intensive care unit; SOFA, Sequential Organ Failure Assessment; SAPS II, Simplified Acute Physiology Score II; OASIS, Oxford Acute Severity of Illness Score; RRT, renal replacement therapy; LOS, length of stay; MBP, mean blood pressure; GCS, Glasgow Coma Scale; AUC, area under the curve; HGB, hemoglobin; PLT, platelet count; ALB, albumin; WBC, white blood cell count; BIL, bilirubin; CRE, creatinine; BUN, blood urea nitrogen; LAC, lactate; APTT, activated partial thromboplastin time; INR, international normalized ratio; PT, Prothrombin time; $\mathrm{Na}$, sodium; $\mathrm{Cl}$, chlorine; $\mathrm{K}$, potassium; $\mathrm{AG}$, anion gap; $\mathrm{FiO}_{2}$, fraction of inspiration $\mathrm{O}_{2}$; $\mathrm{PaCO}_{2}$, partial pressure of carbon dioxide in artery; $\mathrm{PaO}_{2}$, alveolar oxygen partial pressure; $\mathrm{SpO}_{2}$, pulse oxygen saturation.

\section{Data Sharing Statement}

Data were fully available at https://eicu-crd.mit.edu/.

\section{Ethical Approval and Consent to Participate}

This study has been approved by the Institutional Review Board (IRB) of the Massachusetts Institute of Technology (MIT). After successfully accomplishing the National Institutes of Health's (NIH) online training course and the Protection of Human Research Participants
Examination, we had the access to extract data from eICUCRD. All data were extracted by the corresponding author (Certification ID: 28572693). This study was also reviewed by IRB of the First affiliated hospital of Xi'an Jiaotong University and waived the requirement for ethics approval. Given that all patients in this database were deidentified, informed consent was waived.

\section{Acknowledgments}

We are indebted to all individuals who participated in or helped with this research project.

\section{Author Contributions}

All authors made a significant contribution to the work reported, whether that is in the conception, study design, execution, acquisition of data, analysis and interpretation, or in all these areas; took part in drafting, revising or critically reviewing the article; gave final approval of the version to be published; have agreed on the journal to which the article has been submitted; and agree to be accountable for all aspects of the work.

\section{Disclosure}

The authors declare that they have no competing interests.

\section{References}

1. Cecconi M, Evans L, Levy M, Rhodes A. Sepsis and septic shock. Lancet. 2018;392:75-87. doi:10.1016/S0140-6736(18)30696-2

2. Annane D, Bellissant E, Cavaillon J-M. Septic shock. Lancet. 2005;365:63-78. doi:10.1016/S0140-6736(04)17667-8

3. Vincent J-L, Sakr Y, Sprung CL, et al. Sepsis in European intensive care units: results of the SOAP study. Crit Care Med. 2006;34:344-353. doi:10.1097/01.CCM.0000194725.48928.3A

4. Cohen J, Cristofaro P, Carlet J, Opal S. New method of classifying infections in critically ill patients. Crit Care Med. 2004;32:1510-1526. doi:10.1097/01.CCM.0000129973.13104.2D

5. Kim WY, Lee YJ, Yeon Lim S, et al. Clinical characteristics and prognosis of pneumonia and sepsis: multicenter study. Minerva Anestesiol. 2013;79:1356-1365.

6. He X-L, Liao X-L, Xie Z-C, et al. Pulmonary infection is an independent risk factor for long-term mortality and quality of life for sepsis patients. Biomed Res Int. 2016;2016:4213712. doi:10.1155/2016/ 4213712

7. Lambden S, Laterre PF, Levy MM, Francois B. The SOFA score-development, utility and challenges of accurate assessment in clinical trials. Critical Care (London, England). 2019;23:374. doi:10.1186/s13054-019-2663-7

8. Leroy O, Georges H, Devos P, et al. Infective endocarditis requiring ICU admission: epidemiology and prognosis. Ann Intensive Care. 2015;5:45. doi:10.1186/s13613-015-0091-7

9. Wang H, Ye L, Yu L, et al. Performance of sequential organ failure assessment, logistic organ dysfunction and multiple organ dysfunction score in severe sepsis within Chinese intensive care units. Anaesth Intensive Care. 2011;39:55-60. doi:10.1177/0310057X1103900108 
10. Darvall JN, Bellomo R, Bailey $M$, et al. Long-term survival of critically ill patients stratified according to pandemic triage categories: a retrospective cohort study. Chest. 2021;160:538-548. doi:10.1016/j.chest.2021.03.002

11. Benchimol EI, Smeeth L, Guttmann A, et al. The REporting of studies Conducted using Observational Routinely-collected health Data (RECORD) statement. PLoS Med. 2015;12:e1001885. doi:10.1371/journal.pmed.1001885

12. Pollard TJ, Johnson AEW, Raffa JD, et al. The eICU collaborative research database, a freely available multi-center database for critical care research. Sci Data. 2018;5:180178. doi:10.1038/sdata.2018.178

13. van den Boom W, Hoy M, Sankaran J, et al. The search for optimal oxygen saturation targets in critically ill patients: observational data from large ICU databases. Chest. 2020;157:566-573. doi:10.1016/j. chest.2019.09.015

14. Sarkar R, Martin C, Mattie H, et al. Performance of intensive care unit severity scoring systems across different ethnicities in the USA: a retrospective observational study. Lancet Digit Health. 2021;3: e241-e249. doi:10.1016/S2589-7500(21)00022-4

15. Park S-Y, Freedman ND, Haiman CA, et al. Association of coffee consumption with total and cause-specific mortality among nonwhite populations. Ann Intern Med. 2017;167:228-235. doi:10.7326/M16-2472

16. Brotfain E, Borer A, Koyfman L, et al. Multidrug resistance Acinetobacter bacteremia secondary to ventilator-associated pneumonia: risk factors and outcome. $J$ Intensive Care Med. 2017;32:528-534. doi:10.1177/0885066616632193

17. Mörgeli R, Wollersheim T, Engelhardt LJ, et al. Critical illness myopathy precedes hyperglycaemia and high glucose variability. J Crit Care. 2021;63:32-39. doi:10.1016/j.jcrc.2021.01.012

18. Salomão R, Ferreira BL, Salomão MC, et al. Sepsis: evolving concepts and challenges. Braz J Med Biol Res. 2019;52:e8595. doi:10.1590/1414-431×20198595

19. Martin-Loeches I, Povoa P, Rodríguez A, et al. Incidence and prognosis of ventilator-associated tracheobronchitis (TAVeM): a multicentre, prospective, observational study. Lancet Respir Med. 2015;3:859-868. doi:10.1016/S2213-2600(15)00326-4
20. Richardson JD, DeCamp MM, Garrison RN, Fry DE. Pulmonary infection complicating intra-abdominal sepsis: clinical and experimental observations. Ann Surg. 1982;195:732-738. doi:10.1097/ 00000658-198206000-00009

21. Chen L, Deng $\mathrm{H}$, Cui $\mathrm{H}$, et al. Inflammatory responses and inflammation-associated diseases in organs. Oncotarget. 2018;9:7204-7218. doi:10.18632/oncotarget.23208

22. Leligdowicz A, Chun LF, Jauregui A, et al. Human pulmonary endothelial cell permeability after exposure to LPS-stimulated leukocyte supernatants derived from patients with early sepsis. Am J Physiol. 2018;315:L638-L644. doi:10.1152/ajplung.00286.2018

23. Fodor R-Ş, Georgescu AM, Cioc A-D, et al. Time- and dose-dependent severity of lung injury in a rat model of sepsis. Rom J Morphol Embryol. 2015;56:1329-1337.

24. Zhao X, Dib M, Andersson E, et al. Alterations of adhesion molecule expression and inflammatory mediators in acute lung injury induced by septic and non-septic challenges. Lung. 2005;183:87-100. doi:10.1007/s00408-004-2522-3

25. Montgomery AB, Stager MA, Carrico CJ, Hudson LD. Causes of mortality in patients with the adult respiratory distress syndrome. $\mathrm{Am}$ Rev Respir Dis. 1985;132:485-489. doi:10.1164/arrd.1985.132.3.485

26. Klingensmith NJ, Coopersmith CM. The gut as the motor of multiple organ dysfunction in critical illness. Crit Care Clin. 2016;32:203-212. doi:10.1016/j.ccc.2015.11.004

27. Vaishnavi C. Translocation of gut flora and its role in sepsis. Indian $J$ Med Microbiol. 2013;31:334-342. doi:10.4103/0255-0857.118870

28. Von Bültzingslöwen I, Adlerberth I, Wold AE, et al. Oral and intestinal microflora in 5-fluorouracil treated rats, translocation to cervical and mesenteric lymph nodes and effects of probiotic bacteria. Oral Microbiol Immunol. 2003;18:278-284. doi:10.1034/j.1399302X.2003.00075.x

29. Kirton OC, Gore RG, Reid LM, Jones RC. Recurrent episodes of gram-negative bacteremia or endotoxemia change reactivity of preand post-capillary pulmonary segments to angiotensin or free radicals. Intensive Care Med. 1992;18:293-298. doi:10.1007/ BF01706478
International Journal of General Medicine

\section{Publish your work in this journal}

The International Journal of General Medicine is an international, peer-reviewed open-access journal that focuses on general and internal medicine, pathogenesis, epidemiology, diagnosis, monitoring and treatment protocols. The journal is characterized by the rapid reporting of reviews, original research and clinical studies across all disease areas. The manuscript management system is completely online and includes a very quick and fair peer-review system, which is all easy to use. Visit http://www.dovepress.com/ testimonials.php to read real quotes from published authors. 